\title{
Education in Evolution and Science Through Laboratory Activities
}

\author{
Telmo Pievani • Emanuele Serrelli
}

Published online: 26 August 2008

(C) Springer Science + Business Media, LLC 2008

Keywords Bibliography · Evolution · Natural selection · Adaptation · Darwin-Wallace model - Teacher materials . Teacher instruction $\cdot$ Laboratory education $\cdot$ Nature of science

\section{Introduction}

We introduce Investigating Evolutionary Biology in the Laboratory (henceforward IEBL) with these words by the editor:

Evolution is the unifying framework for the science of biology. [...] It likewise demands an equally dominant role in biology education. [...] Simultaneously it is the most important, the most misunderstood, and most maligned concept in the syllabus - if it even appears in the syllabus (Investigating Evolutionary Biology in the Laboratory, by William F. McComas (ed.). Dubuque (Iowa): Kendall-Hunt, 2006. pp. vi $+388 . \mathrm{s} / \mathrm{b} \$ 41,99$ ).

IEBL is a guide for biology teachers in search of good ideas to enhance their teaching of evolution, "designed to build on what textbooks contain, not to replace them" (McComas, p. 3).

The manual features two parts. The second part, occupying around two-thirds of the book, is a repertoire of 32 laboratory activities for teachers to pick up, perhaps modify,

Invited review of McComas W. (ed.). Investigating evolutionary biology in the laboratory. National Association of Biology Teachers (NABT), Kendall-Hunt, 2006.

T. Pievani $\cdot$ E. Serrelli $(\bowtie)$

"Riccardo Massa" Department of Human Sciences,

University of Milan Bicocca,

Piazza dell'Ateneo Nuovo,

1-20126 Milan, Italy

e-mail: emanuele.serrelli@unimib.it and integrate in a high school or college curriculum. But, there is more in IEBL than ready-made activities: Especially through the essays collected in the first part, this book can help readers for better understand evolution, prepare themselves to the teaching of evolutionary biology, and even reorganize science education in a way that deeply involves teacher, students, and science, eventually leading to the persuasion that laboratory activities of a certain kind are a superior way of teaching evolution and science.

\section{Investigating the Darwin-Wallace Model}

The second part of IEBL consists in a selection of papers published mainly in The American Biology Teacher from 1966 to 2004 and adapted by William F. McComas (Chair of Science Education, University of Arkansas). The collection also contains some contributions originally written for IEBL.

We copied Table 1 just to give a glance at the range of arguments (35 "evolution principles" identified by McComas) covered by the 32 laboratory activities that come organized into six sections: "Examining the Evidence for Evolution," "Using the Tools and Principles of Evolution," "Variation and Adaptations within Species," "Biotic Potential and Survival," "Simulating Natural Selection," and "The New Evolutionary Synthesis."

As can be seen from the table, there is no rigid sectionprinciples correspondence: For example, "systematics and classification" is a key principle in the "Evidence of Evolution" section, but it comes again in "Using the Tools and Principles" and in "The New Evolutionary Synthesis." Also, we have found some activities deeply involved with principles not signaled in the table. This is the case with the "natural selection" principle, present in many activities outside the dedicated 


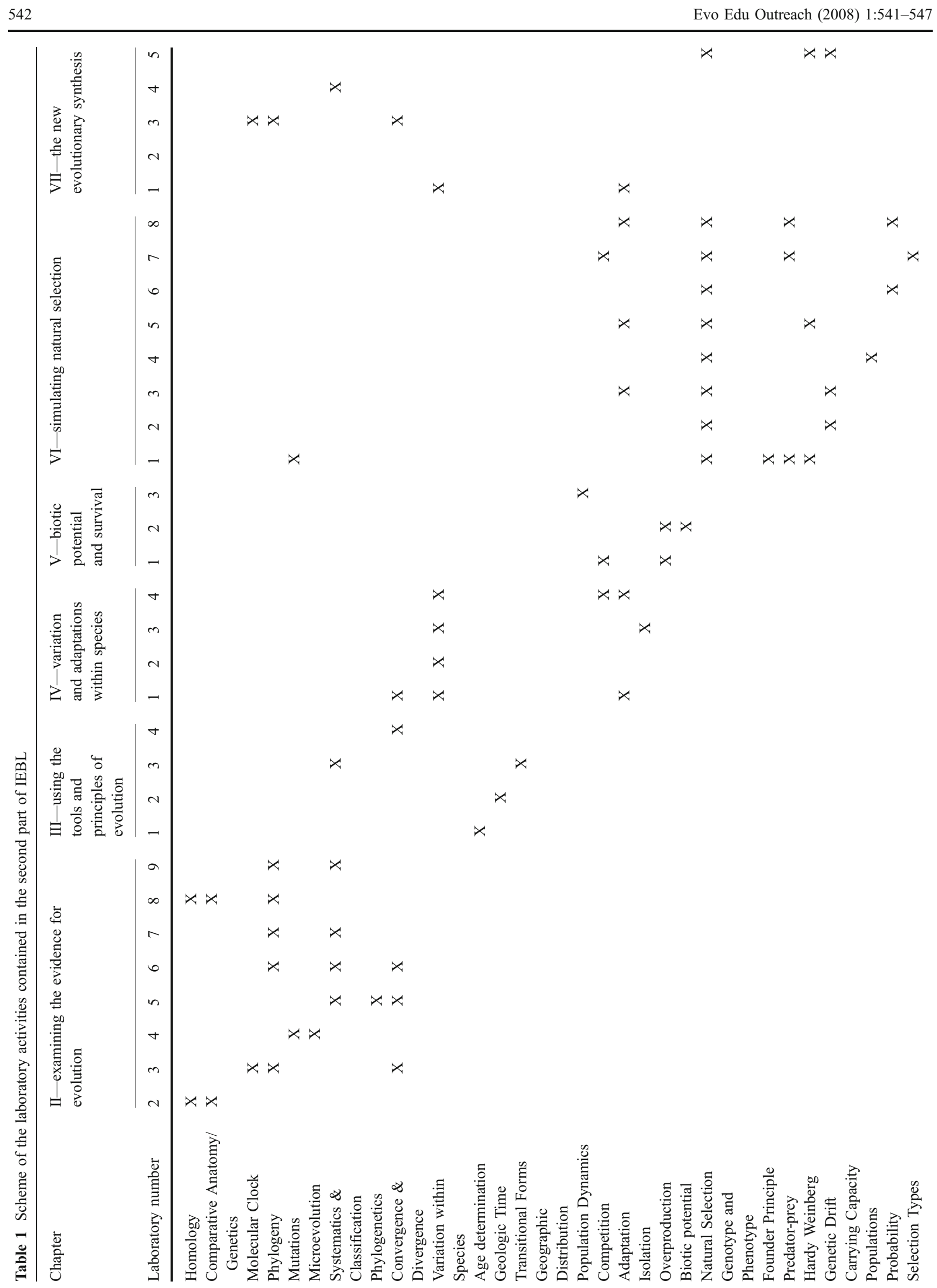

黑 Springer 


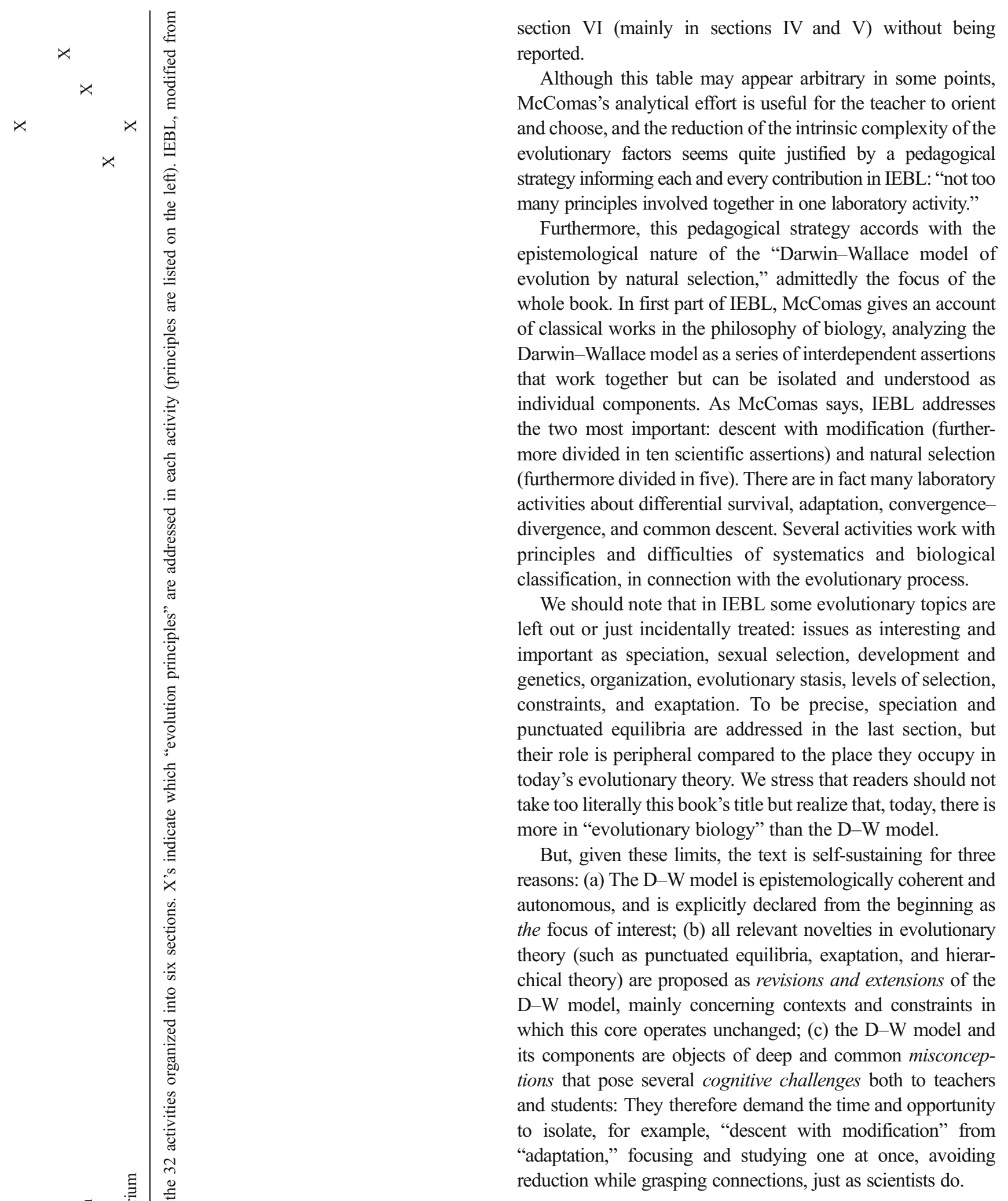

\section{Activities Overview}

We think that the 32 activities collected in IEBL refute two possibly fallacious ideas about biology teaching, i.e., (a) 
laboratory experiments about evolution would be impossible, since evolution is a matter for historical and not experimental science, featuring unrepeatable and irreproducible events that happen in large space and time scales; (b) even if possible, setting up a biological laboratory implies expensive, sophisticated, sometimes hazardous equipment, or extraordinary experimental conditions.

Well, every activity collected in IEBL has a "Materials" paragraph, and the great part of them just require few everyday objects: peanuts, M\&M's, seeds, pinecones, cereals, varieties of beans, apples, Jelly Belly beans, tomatoes, pears, leaves; markers, pencils, stickers, tape, meters and rulers, paper punch; pliers, fine point forceps, knives, spoons, straws, forks; plastic containers or bags, colored toothpicks, wooden matches, nail polish, coffee cans; colored paper chips, construction paper, and pieces of fabric.

With the help of rules to follow, dice, a Yahtzee game, playing cards, calculators, etc., students often become part of the mechanism to be learned: They turn into predators, timescale indicators, "reproductors," and so on, favoring the emergence of solutions that make sense for them. This is consistent with a constructivist view of learning: "students can only assimilate new information by generating personal understanding out of their own experiences" (McComas, $\mathrm{p}$. 10). Sometimes students themselves can also be the direct object of observation, as humans are inside evolutionary processes and bear their traces.

In some (actually, not many) cases, materials can be usefully enriched or switched out for actual and direct biological and paleontological materials: An essay is dedicated to 'Designing Fossils Collections for 'Hands-On' Evolution Laboratories" (II-1, by James E. Platt, 1999), and another one gives advice for observing and labeling skeletal partshowever, real skeletons are allowed to be replaced by illustrations (II-2, by Robert A. Coler, 1966). In summary, direct experiences with such materials seem to be an advisable integration into usually self-sufficient laboratory activities. Two IEBL activities involve culturing and breeding of organisms (Drosophila, worms, and plants), two make use of microscopes, and two involve chemicals, biochemicals, and relevant experimental apparatus. It must be said that there is no direct link between the technological level of the apparatus and the cognitive value of the experience. On the contrary, as we will show below, the latter two activities appear to be the less coherent with IEBL philosophy and could probably be the less effective in terms of learning.

Many activities are well suited to be realized inside the classroom; others can be usefully conducted outdoors: in a football field, at the zoo, or in a park.

Printed drawings, worksheets, figures, transparencies, and handouts are provided as veritable working materials for students to work on with pen, colors, scissors, and glue. There are few test-like questions or demonstrative illus- trations. Often, it could be more interesting to give students an empty graph sheet than show them a chart! Essential data for conducting the laboratory are delivered to the teacher mainly in form of tables, schemes, and the "Background Information" paragraph in each activity.

Two activities make use of very interesting Internet resources: the same databases and programs that are used by scientists for comparing genetic sequences.

\section{Teachers and Students Working Together in Science}

The first third of IEBL, named "Foundations of Evolution Education," does contain a series of synthetic but penetrating essays by McComas and colleagues. One of the main standpoints of their thinking could be so expressed: There is and there must be a deep connection between what happens in laboratory instruction and what happens in 'real' science. An instance of that could be the kind of "modular isomorphism" we have seen between the Darwin-Wallace model and the possible laboratory activities, but essays point out many more insightful indications for designing and conducting laboratory activities. Some examples are: Always place laboratory before explanatory sessions; use laboratory to introduce, not just verify concepts; provide thought-provoking challenge questions to students in the briefest fashion possible; allow students to make real choices during the investigation; and so on.

In the approach called "learning cycle," referred to in some passages of the first part of IEBL (see in particular the essay by Anton E. Lawson), the teacher's introduction of new terms and concepts is always preceded by the students' "hands-on" explorations, observations, descriptions, tentative definitions, and is often followed by the endeavor of concept application in new and different domains. As students may learn in their laboratory "learning cycles," "debate, rethinking, and a cycle of verification are important elements of the scientific endeavor and, as such, are inherent in healthy scientific discourse" (McComas, p. 21).

Laboratory activities should accurately portray the nature of science (NOS). This is a central issue throughout IEBL (there is also a specific contribution about that by Michael P. Clough). In well-designed and well-conducted laboratories, students construct new meaningful concepts and generate "if...and...then...therefore" arguments and hypotheticodeductive inferences, managing "ideas that work extremely well at explaining the natural world in naturalistic terms we can understand, making accurate predictions, and guiding further empirical research" (Clough, p. 72), in other words, experiencing the way science comes down to patterns that are naturalistic, testable, and always open to revision.

Much can be done to make students' activities even more similar to scientists' work. For example, since "scientists 
rarely do investigations where the solution is assured within an hour or so" (McComas, p. 8), activities can be expanded in time and space beyond the classroom by assigning daily or weekly observations or by distributing complementary research tasks to different and distant groups (networking).

Avoiding any kind of ideology, emphasizing curiosity and the utility of knowledge, laboratory activities invite students to explore problems without demanding preventative "declarations of faith" of them, in respect to their own (especially religious) beliefs.

Laboratory activities can improve students' abilities to inquire, work in teams, select, present, discuss, and comment on results.

Entering directly into the interdependence between theoretical context, scientific practice, and discoveries, students can also explicitly discuss NOS with the teacher's help, addressing some misconceptions - pointed out in essays by Clough and others - according to which scientific ideas and models would be "exact copies of the natural world," a theory would be "just a theory," susceptible to become a law if and when it is "verified," and so on.

"Working inside science" in the lab can also give students the opportunity to appreciate and accept that "well-established scientific knowledge is not fair, nor is it decided democratically":

students need to be made aware that the scientific community, not public opinion polls, individual scientists, or small groups of scientists decide what is good science (Clough, p. 74).

Students can reflect as well upon issues on the growth of science:

Perhaps the most counterintuitive notion that comes from the NOS is the well-supported view that unresolved puzzles and seemingly refuting evidence do not always result in rejection of a scientific idea (Clough, p. 76).

A laboratory can also be the right context to present the human dimension of science instead of the purified versions of discovery often found in textbooks: The laboratory can contextualize history, giving the teacher the chance to tell stories from the history of science in a more vivid way and grounded on the students' direct experience (as an example, there is in IEBL a whole essay by McComas concerning textbook myths and misconceptions about Darwin's work and life).

Laboratory activities must be designed in coherence with the nature of science. The NOS and the laboratory will then form a circular dynamic: While the NOS helps students make sense of their laboratory experiences, the latter in turn helps students to learn about the NOS.

But this "working in science" can be a useful training for the teacher as well. As McComas points out in a review of the US school system, some rigid, dogmatic, authoritarian positions taken by teachers in the face of evolution may come from perplexities not about content but about the nature of science. Indeed, some studies show that a deep understanding of the NOS reduces teachers' anxiety toward the teaching of evolution, while today few biology teachers appear to be prepared to argue effectively against intrusions into the biology curriculum. To understand, for example, "why ID is not science and why biological evolution is a sound scientific theory requires a sophisticated understanding of the nature of science" (Clough, p. 70). Some misconceptions regarding the NOS can consequently occur just by teachers' behavior or choice of language.

Once again, "evolution challenges teachers and their students alike.” In IEBL, teachers can find precious clarifications and reassurances:

The testable deductions that follow from evolutionary theory make clear that it is science. The evidence in support of these deductions make clear that biological evolution has not been falsified (Clough, p. 76, our italic).

Evolution as change through time has occurred; scientific debates are aimed at "fine-tuning" our understanding of the mechanisms and singular cases. IEBL also gives precise references to the First Amendment of the US Constitution and Supreme Court decisions stating that a state, school, or district cannot ban the teaching of evolution, cannot require equal time for creationism or creation science, or have a disclaimer that singles out evolution from the science curriculum.

\section{Teacher in the Lab: A Portrait}

We suggest that teachers reading IEBL can find support and new ideas for their practice but also undertake a selftraining that goes right through laboratory instruction.

In IEBL, William McComas and colleagues draw the portrait of a teacher who takes science and its nature seriously: First, by putting the pervasive framework of biology, namely evolution, right at the center of biology teaching; starting by the manual title, we see a teacher investigating even more than teaching in some traditional sense of "transmitting knowledge"; this teacher always tends to be brief: He or she must constantly refrain from giving notions to students (that would often be answers to never-asked questions!); she has the key role to provoke, to challenge students, and to suggest to them activities for finding answers themselves; according with the "degree of openness" of the particular laboratory, he allows real choices; she has a facilitating style: rather than interrupting and correcting students, she helps to spread students' good ideas and strategies in the classroom and to find new ones. Such a teacher's work is renewed and 
enriched year after year by new and unexpected results. Of course, he must find solutions for an authentic and honest assessment, respecting the nature of the laboratory activity.

Well, it is evident-and explicitly declared by the authors - that such a teacher must come prepared for the laboratory experience: He or she has to be "highly skilled." But while it is true that laboratory requires much of the teacher, we ought to say that laboratory can help the teacher to construct his own professional identity. So we advise teachers not to wait to become "highly skilled enough" to start using laboratory activities; on the contrary, they can use laboratory activities to construct their competence. And even though the first trials might not be so good, it will be another level of discovery and enhancement.

To end our review, to stress once more the importance of "Foundations of Evolution Education" (henceforward FEE), the first part of IEBL: In order to conduct meaningful and effective activities, teachers need to take hold of the general indications therein concerning evolution and the laboratory. This is necessary because a good experimental apparatus can still be ineffective or even confirm misconceptions due to the teacher's performance (style, procedure, language, etc.). On the other hand, a prepared teacher familiar with FEE contents can effectively modify and use activities that are not correctly conceived or exposed.

\section{Using Activities Critically: Two Examples}

Take for example "Comparative anatomy as evidence of evolution" by Robert A. Coler (II-2, 1966), in which students are asked to find and mark homologous features across various skeletons of different species:

As students identify and observe homologous structures in different groups of animals, help them to see that their differences arose as a result of gradual change over time. Homologous structures in amphibians, birds, reptiles, and mammals, for example, developed over hundreds of millions of years - the time it took for each of the respective groups of animals to evolve (Coler, p. 127, our italic).

Just by this quotation in the light of FEE, we can see two problems here: (a) Animals are referred to as "related" since the very beginning of the activity: an evolutionary scheme is strongly presupposed, so the activity appears to be somewhat apologizing, aiming to convince that there is a change though time, whereas according to FEE indications, the students themselves should hypothesize or at least evaluate alternative explanations; (b) the activity is presented as if it would be a discovery of the passage between existing animals, with a progressionist accent that maybe reflects conceptions of 40 years ago (this is the oldest activity in the collection, 1966).

A teacher who is familiar with FEE can easily recognize and overcome these limits and use Coler's indications in a more appropriate way. For the first problem, incidentally in this case, he or she can find a good counter-example in "A Scientific Approach to Teaching about Evolution and Special Creation" by Anton E. Lawson (1999, I-7), presented in FEE as a paradigmatic example of a "learning cycle approach." This activity:

...raises the key scientific question and presents the major alternative explanations/theories that have been proposed in the past to answer the question. This approach then challenges students to gather evidence from the fossil record and analyze that evidence using critical thinking skills to decide for themselves which explanation (or explanations) represents the best answer to the question raised (Lawson, p. 82).

A detailed guide for the teacher follows, concerning how to help students generate hypothetico-deductive arguments for testing the competing theories (with transcriptions of conversations as examples).

The second problem is another confirmation, if needed, that teachers have to be conscious and up-to-date in selecting and using laboratory activities. The last 40 years of discussions in biology and epistemology have brought to light cognitive challenges and misconceptions (some of which are mentioned in FEE), and also scientists have changed their language, refined and redefined old problems and descriptions. In this particular case, two tendencies are relevant: (a) The preference for linear reconstructions of evolutionary stories has given way to branching models, making scientists extremely careful in constructing easy long-span linear sequences such as amphibians-birds-reptiles-mammals, now preferably seen as "evolutionary trends;" (b) in many cases, it has been necessary in science communication to state more clearly that, in evolution, the word "derivation" traces back to a common ancestorship: In short - to cite a widespread misconception - humans do not derive from actual apes, but both groups descended from a common ancestor.

Less subtle critiques can apply to a much more recent activity: "Antigen-antibody interaction" by Mary Culp (2000, II-3), in which

...students will test to see whether antigens and antibodies across several species react with each other. Using these data, evolutionary relationships between the chosen species will be constructed (Culp, p. 128).

In this activity, students make use the Ouchterlony test (1973), which requires a kit with instruments, chemicals, and biomaterials such as agar, $\mathrm{NaH}_{2} \mathrm{PO}_{4}, \mathrm{NaCl}$, antisera, 
hot and boiling water baths, incubator, micropipettes, and so on, with an extensive preparation by the teacher.

It seems to us that this activity, as it is presented, is an example against many of the principles exposed in FEE: In contrast with the "emphasis on postlab discussions" principle, students need a very large amount of background information (no less than specificity of antibodies, taxonomic relationships between animals, and the molecular clock theory) in order to really understand what they are doing; they do not really discover anything nor can they make real research choices or predictions; on the contrary, they are guided step-by-step in demonstrating what is known by the start- this is also clear from the "troubleshooting" section that explains to the teacher what to do if the results are not exactly as expected; students have to be very confident in evolution and science because what they can actually see are just indirect phenomena of a little part of the whole process: roughly, white precipitates formed by liquids poured from labeled bottles.

Here we confront a very traditionally conceived laboratory. Similar problems are found for example in "Evolution in the Laboratory: Biocide Resistance in E. Coli" by Rex A. Hossler and Charles W. Welden (2003, II-3), in which students should "discover" that bacteria rapidly evolve resistance to triclosan. Again, the explanation is given, and the lab serves as a possible demonstration; high level of previous knowledge and preventative confidence are requested; students execute orders (up to seven per page, plus teacher interventions); they do not elaborate strategies, no questions: There is a precise procedure, the sense and endpoint of which is held by the teacher.

Working with true scientific materials could be considered in any case pedagogically valuable or necessary for students in order to really understand science. But, in coherence with IEBL and FEE, we believe that such activities could also have scarce learning effects unless they come after long series of other experiences through which students actively come to grasp the many elements constituting the "background knowledge," possibly getting themselves to the question and acquiring instruments with which even mistakes and unexpected results can be fecundly discussed. Paradoxically, such a huge previous work would make the final "hands-on" laboratory activity just a complement allowing students to become familiar with scientific technical equipment.

\section{Conclusions}

In conclusion, we must stress that IEBL contains a lot of smart and very useful activities: Issues about DNA can be effectively treated with paper and scissors (VII-3) or the validation of phylogenies using the degree of variation found in one or few traits across different species can be addressed through classic activities such as the Caminalcules (II-5, II-6, and VII-2). Theoretical and philosophical reflections found in the first part of IEBL are equally, if not more, important than the activities in the second part, not to be considered as "copy-and-deliver," "ready-made" activities to add to the standard curriculum. Reflecting critically upon the main problems concerning laboratory instruction and evolutionary biology, teachers can really improve their approach, conduct activities in a meaningful and effective way, even evaluate critically the proposals contained in IEBL, and build new ones. 